\title{
Contribution of Perceived Rearing to Depression: The Role of Cognitive Patterns as a Mediator
}

\author{
Toshinori Kitamura* and Nao Tanaka
}

Kitamura Institute of Mental Health Tokyo, 101 Akasaka 8-5-13, Minato, Tokyo, Japan 107-0052

\begin{abstract}
To test possible mediation of the effects of the perceived parenting in childhood on depression, university students $(\mathrm{N}=258)$ were examined using a cross-sectional questionnaire survey. A path model indicated that (1) the effects of dysfunctional attitude (rated by the Dysfunctional Attitude Scale) on depression (rated by the Center for Epidemiologic Studies Depression Scale) was mediated by automatic thoughts (rated by the Automatic Thought Questionnaire-Revised), (2) the effects of perceived rearing on depression were mediated by dysfunctional attitudes and automatic thoughts, and (3) perceived rearing was influenced by the gender of the student. These findings suggest that the well-studied link between childhood experiences and adult depression are mediated by depression-related cognitive styles.
\end{abstract}

Keywords: Perceived rearing, depressogenic cognition, automatic thought, dysfunctional attitude, vulnerability for depression.

\section{INTRODUCTION}

Beck's [1] theory on and practice of depression constituted a paradigm shift, with depression-specific cognitive distortions causing depressed mood rather than vice versa. His cognitive theory maintains that clinical depression is triggered when negative life events are followed by the appearance of a specific negative condition: the presence of automatic thoughts. These thoughts take the form of overly negative beliefs about oneself, one's world, and one's future - the negative cognitive triad.

This process, however, does not take place in every individual. According to Beck's cognitive diathesis-stress theory, it occurs when individuals possess dysfunctional schemata containing attitudes on the theme such as loss, inadequacy, and failure. These include various enduring beliefs, such as predicating happiness on being perfect or on the approval of others. These dysfunctional attitudes are not activated until one encounters a negative life event and the ensuing automatic thoughts.

Empirical studies on dysfunctional attitudes, often using the Dysfunctional Attitude Scale (DAS) [2], have shown that people with high scores on the DAS are more likely to be depressed [3-10]. Higher DAS scores predicted the onset of depression in a study with a longitudinal design [11]. Individuals with depression score higher on scales assessing automatic thoughts, for instance the Crandell Cognitions Inventory (CCI) [12] and the Automatic Thoughts Questionnaire (ATQ) [13], than do individuals without depression [4, 12, 14-18], nonpsychiatric medical patients [17, 19], or

*Address correspondence to this author at the Kitamura Institute of Mental Health Tokyo, 101 Akasaka 8-5-13, Minato, Tokyo, Japan 107-0052;

Tel: +81-3-6804 5662; Fax: +81-3-6804 5662;

E-mail: kitamura@institute-of-mental-health.jp remitted depressed patients $[4,14,17]$. Patients also score higher on these scales while depressed than they do after they have remitted $[4,15,16]$. Patients with depression scored higher on the ATQ than did heterogeneous nondepressed psychiatric control samples [4, 17, 19, 20]. Automatic thoughts were correlated with other measures of depression [21].

Despite the above findings, few studies have investigated why some people become vulnerable to depression by developing dysfunctional attitudes while others do not. The origin of depressogenic cognitive styles may date back to the early years of life, with an individual's acquisition of these styles determined by their early psychosocial environment.

The rearing attitudes and behaviours of parents are associated with the psychological development of their children and the onset of adult mental disorders (for review see [22]). Many investigations have reported that exposure to inadequate parenting (usually low care or overprotection) is associated with increased risk of depression in adulthood [2338]. One argument against the hypothesis that perceived rearing and depression are linked is the time lag between the rearing experiences as a child and the onset of depression as an adult. Since the first report of Parker and colleagues, there have been only a few investigators who have investigated the variables that mediate the two.

Whisman and Kwon [39] hypothesised that cognitive vulnerability factors mediate the relationship between parental representation and depression. They found that among 150 college students, parental low care and overprotection were correlated with depression but that these associations were no longer observed after controlling for dysfunctional cognitive styles. They therefore claimed that cognitive styles mediated the link between the parental representation and depression. Their study used either regression analyses and 
correlation coefficients. Proof that cognitive styles mediate the link between perceived rearing and depression may be better obtained using structural equation modelling (SEM) because there are more than one possible mediator in our design [40]. Our study of Japanese university students employs SEM to examine the relation between perceived rearing, depressogenic cognitive styles, and depression.

\section{METHOD}

\section{Participants}

Students of two universities in Kumamoto were solicited for participation in the present study. A total of 376 questionnaires were distributed. Only one student declined to participate. We excluded cases with missing values of the questionnaires we analysed in this study. This resulted in 259 cases. We also excluded one individual because their age was over 25 years (aged 56). Thus, the final sample used for further analysis consisted of 258 cases (169 men and 89 women). Age did not vary with gender (men: mean age, 19.4, SD 0.7; women: mean age 20.0, SD 1.0).

\section{Measures}

\section{Depression}

Center for Epidemiologic Studies Depression Scale (CES-D) [41] is a self-report scale consisting of 20 items. It was designed to cover the main features of the depressive syndrome as identified by clinical features and factor analysis. Radloff [41] reported that the scale was developed for use in studies of the epidemiology of depressive symptoms in the general population. Reliability and validity of this scale were confirmed [41].

\section{Automatic Thought}

Automatic Thoughts Questionnaire-Revised (ATQ-R) [20] is a 40-item self-report measure of an individual's automatic thoughts. Each item is rated on a 5-point scale ranging from 1 ("not at all") to 5 ("all the time"). The ATQ-R include two subscales, the ATQ-R negative (30 items) and the ATQ-R positive (10 items). The ATQ-R negative consists of negative self-talk statements whereas the ATQ-R positive consists of positive self-talk statement. The scores of ATQ-R positive items are reversed and added to those of the TTQ-R negative to yield the ATQ-R score. Possible score of the ATQ-R, thus, range from 40 to 160, with higher scores indicating a greater number of negative automatic thoughts. We translated the ATQ-R into Japanese with the original author's permission and it was back-translation into English to verify the wording by him.

\section{Dysfunctional Attitude}

Dysfunctional Attitude Scale (DAS) [2] is a self-report measure of the beliefs or schemata that underlie the characteristic cognitive content of depression as outlined by Beck's cognitive theory of emotional disorders [1]. The DAS consists of 40 items rated on a 7-point Likert scale ranging from 1 ("totally agree") to 7 ("totally disagree"). Examples of items include, "My value as a person relies greatly on what others think of me," and "If I fail at work then I'm a failure as a person." Higher scores indicate more maladaptive think- ing. The DAS shows good internal consistency and adequate psychometric properties [2].

\section{Perceived Rearing}

Parental Bonding Instrument (PBI) [42] is a self-report questionnaire used to describe a parental attitude toward the subject. There are 12 Care items and 13 Overprotection items. The 25 items were scored on a 4-point Likert scale, from 1 ("very unlikely") to 4 ("very likely"). Each composite score was used in the present study; higher scores reflect higher care or higher overprotection experiences. The PBI has been reported to have good reliability [42]. The PBI was translated by Kitamura and Suzuki [43], with backtranslation into English to verify the wording. The validity of the instrument was confirmed by a high concordance of each parent's rating between the assessment generated by the student and that generated by the parents themselves.

\section{Data analyses}

For descriptive purposes, means and SDs of all the variables used in this study and correlations between them were calculated.

A hypothetical model was constructed using structural equation modelling (SEM) in order to assess direct and indirect effects between variables and an indication of the overall fit of the initial model (Fig. 1). We posited that (1) Care and Overprotection scores of both fathers and mothers would be influenced latent variables: Paternal and Maternal Parenting, respectively; (2) Both Paternal and Maternal Parenting would influence the scores of the DAS, ATQ-R, and CES-D; (3) the DAS scores would influence both the ATQ-R and CES-D scores; (4) the ATQ-R scores would influence the CES-D scores; (5) the gender of the participant $($ men $=1$; women $=2$ ) would influence the scores of Paternal and Maternal Parenting, and the DAS, ATQ-R, and CES-D scores; (6) the error variables of the Paternal and Maternal Parenting would share covariance; and (7) the error variables of Care and Overprotection among fathers and mothers would share covariances between the two parents.

Because our interest was in possible mediation of the effects of the perceived raring in childhood on the current depression by dysfunctional attitude and automatic thoughts, we examined two revised models in which the ATQ-R was deleted as well as both the ATQ-R and DAS were deleted and compared them with the original model [44].

The fit of each model with the data was examined in terms of chi-squared (CMIN), goodness-of-fit index (GFI), adjusted goodness-of-fit index (AGFI), comparative fit index (CFI), and root mean square error of approximation (RMSEA). According to conventional criteria, a good fit would be indicated by CMIN/df <2, GFI > .95, AGFI $>.90$, CFI > .97, and RMSEA < .05; an acceptable fit by CMIN/df $<3$, GFI > .90, AGFI $>.85$, CFI > .95, and RMSEA < .08 [45]. Statistical analyses were conducted using SPSS 19.0 and structural equation modeling with AMOS 19.0 was used to construct and determine the best-fitting model.

\section{Procedure}

We handed out the questionnaires in class and collected them once students had finalized their responses. Infor- 


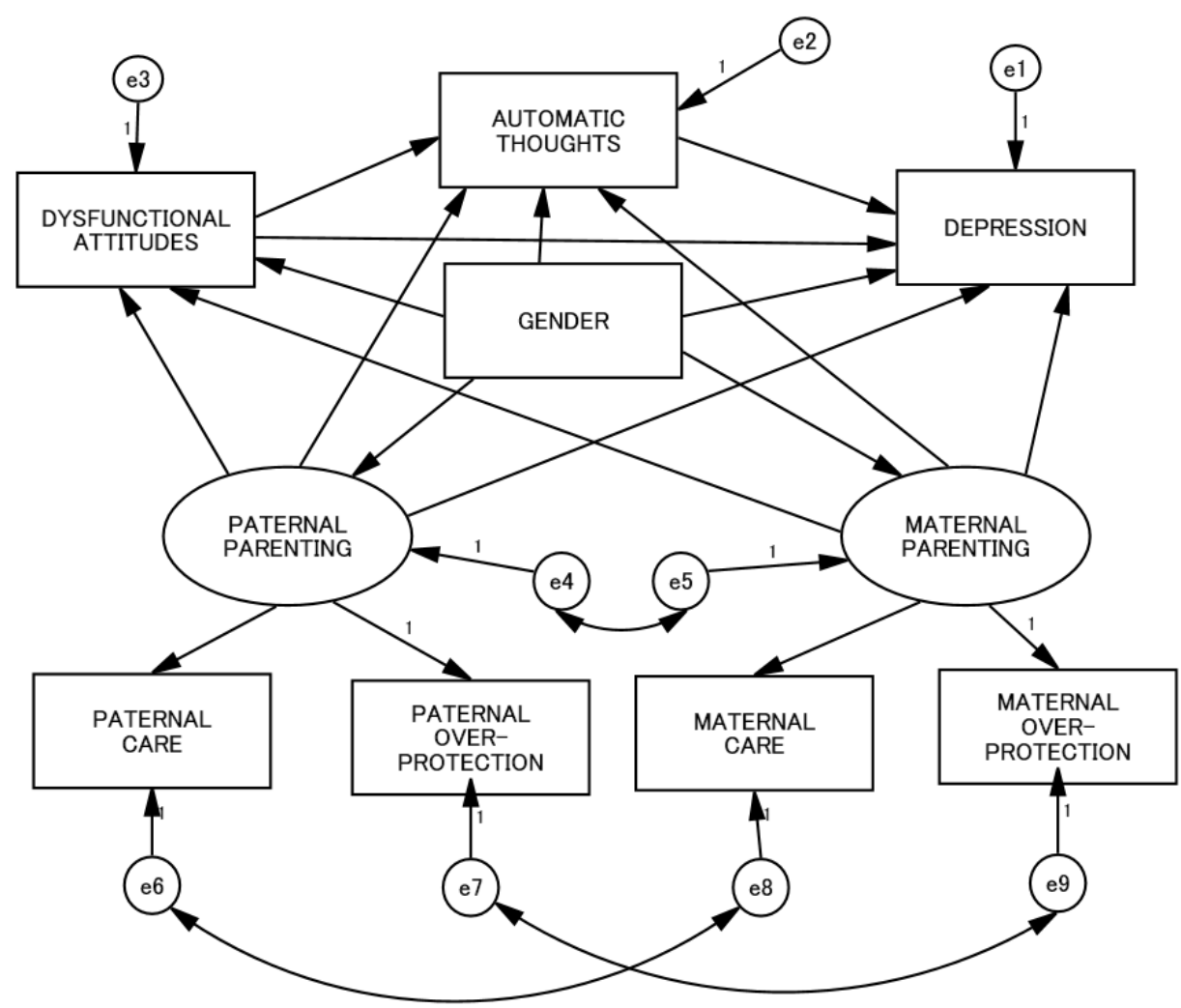

Fig. (1). Hypothesized path model.

Table 1. Means, Standard Deviations, and Correlation Coefficients of CES-D, ATQ-R, DAS, and PBI Subscales (N = 258)

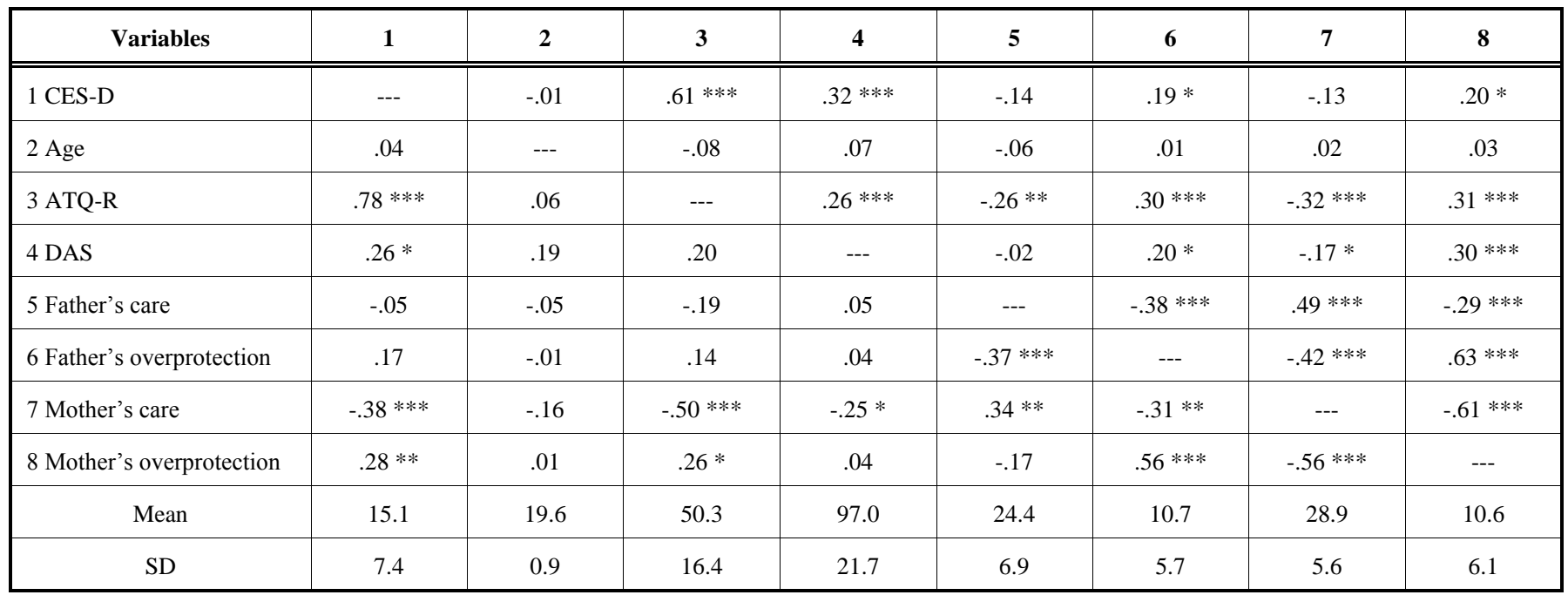

$* p<.05, * * p<.01, * * * p<.001$. CES-D = Center for Epidemiologic Studies Depression Scale; ATQ-R = Automatic Thoughts Questionnaire-Revised; DAS $=$ Dysfunctional Attitude Scale; Figures upon the diagonal represent male students whereas figures under the diagonal represent female students.

mation about informed consent was presented in the questionnaire, and consent was obtained from all subjects. This research project was approved by the Ethical Committee of Kumamoto University Graduate School of Medical Sciences.

\section{RESULTS}

\section{Descriptive and Bivariate Data}

Descriptive data on all of the variables are shown in Table 1. The bivariate relations among the variables are shown among men and women separately. CES-D, ATQ-R, and DAS scores were positively correlated with each other except for the correlation between ATQ-R and DAS in women. Maternal low Care/Overprotection was associated with ATQ-R in men and women. It was also associated with DAS in men and CES-D in women. Maternal Care was negatively correlated with DAS in women whereas maternal Overprotection with CES-D in men. Paternal low Care/Overprotec-tion was correlated with ATQ-R in men. Also in men, paternal Overprotection was correlated with CES-D and DAS. 
Testing the Model: Structural Equation Modeling Procedures

The original model fit the data well: $\mathrm{CMIN} / \mathrm{df}=1.33$, GFI $=.991$, AGFI $=.954, \mathrm{CFI}=.996$, RMSEA $=0.036$ (90\% CI $0.000-0.090)$. The final model (Fig. 2) showed that depression was predicted by automatic thoughts and dysfunctional attitudes, and that automatic thoughts were predicted by dysfunctional attitudes, $2^{\text {nd }}$ Maternal Parenting (low Care/Overprotection). Dysfunctional attitudes were predicted by Maternal Parenting (low Care/Overprotection). Students' female gender negatively predicted both parents Parenting (low Care/Overprotection).

It seemed that the maternal perceived rearing had indirect effects upon depression through dysfunctional attitude and automatic thought. Hence we examined whether this was mediational effect by deleting the ATQ-R and both the ATQ-R and DAS from the original model. When the ATQ-R was deleted from the model, the model fit the data well; $\mathrm{CMIN}=8.052, \mathrm{df}=5, \mathrm{GFI}=.991, \mathrm{AGFI}=.951, \mathrm{CFI}=.992$, RMSEA $=0.049$. The path coefficient from the DAS toward CES-D was increased from .14 to .25. When both the ATQ$\mathrm{R}$ and the DAS were deleted, the path coefficient from the maternal parenting to depression was substantially increased from -.04 (non-significant) to $.22(p=.036)$ with CMIN = $5.624, \mathrm{df}=3$, GFI $=.993, \mathrm{AGFI}=.949, \mathrm{CFI}=.992$, RMSEA $=.058$. Therefore the effect of the maternal rearing on depression was interpreted as being mediated by dysfunctional attitude and automatic thought.

The final model (Fig. 2) had a few non-significant paths. Therefore we restrained non-significant paths to zero one by one. The increase in CMIN was not significant when the path was restrained to zero from the paternal parenting toward depression $(\mathrm{CMIN}$ increase $=0.102, \mathrm{NS})$; from maternal parenting toward depression $(\mathrm{CMIN}$ increase $=0.962)$; from paternal parenting toward dysfunctional attitudes $(\mathrm{CMIN}$ increase $=0.867)$; from paternal parenting toward automatic thoughts (CMIN increase $=2.969)$; from gender to depression $(\mathrm{CMIN}$ increase $=1.023)$; from gender to automatic thought $(\mathrm{CMIN}$ increase $=0.180)$; and from gender to dysfunctional attitudes $($ CMIN increase $=2.306)$. These respecification of the model indicated that simpler model can represent the data not worse than the original model.

\section{DISCUSSION}

As expected from the past literature, the present study demonstrated that the effect of depressogenic schema (rated by the DAS) on depression (rated by the CES-D) was partially mediated by automatic thoughts (rated by the ATQ-R). The direct influence of depressogenic schema on depression was very small. Bivariate correlations showed that maternal low Care and high Overprotection during childhood affected adult depression. However, these correlations ceased to be significant in the present path models. Thus, parental care and overprotection influenced, if anything, only depressogenic schema and automatic thoughts. The direct paths from PBI subscale scores towards depression lost their significance with further refinement of the path model. The totality of our findings may shed light on the time lag between parental rearing during childhood and the possible development of depression over a decade later, following exposure to stressful life events, and on the reasons why cer-

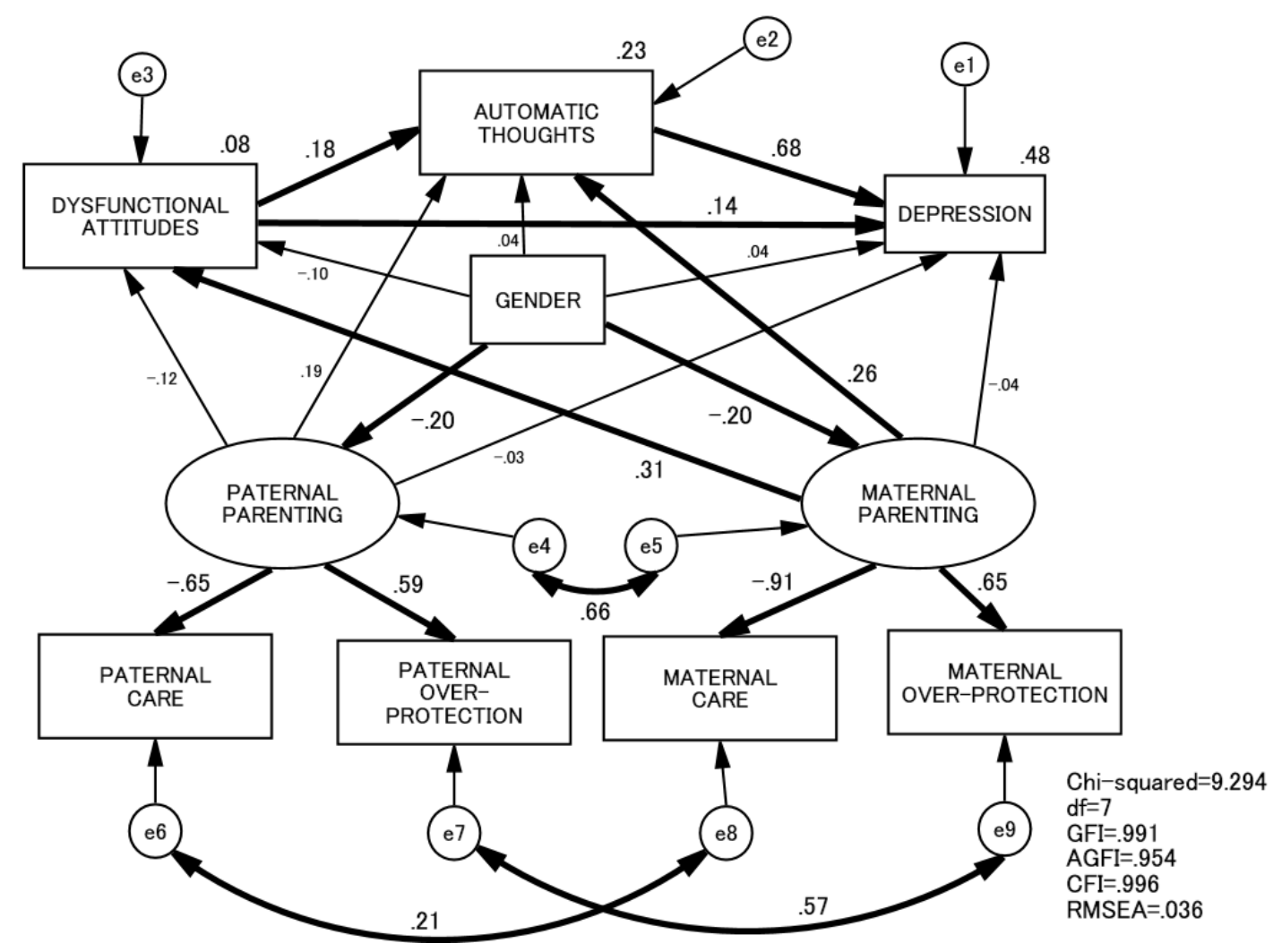

Fig. (2). Final path model. 
tain individuals develop depression as a result of depressionrelated cognitive characteristics.

Men and women did not differ substantially in terms of correlations between variables used in this study except for a few such as maternal overprotection vs. DAS. In a SEM gender showed significant paths only toward paternal and maternal parenting.

The current study has several limitations. First, it is difficult to make generalizations based on this study due to the limitation of the sample population, which consisted of students from only two universities. Clinical populations should be studied using the same methodology before extrapolating the data to non-clinical settings.

Second, this study is based on a cross-sectional design. It may be that depressed mood leads students to recall their parents as uncaring and as overprotective. However, repeated measurements of parental representations have shown that memories of parental behaviour are highly stable across clinically significant changes [46]. Perceived rearing may be confounded by age [47] but the age range of the students in this study was negligible. A low degree of agreement between parents and children in reports of parental behaviors has been demonstrated [48]. Thus, it is feasible that those students with depressogenic schema, even though not actually depressed, are more likely to report their parents as having been less caring and more overprotective. Future replication studies may require the use of parents' reports about their spouses' behaviors and attitudes towards the child, because there has been shown to be more agreement between parents' assessment of each other than between parent and child [43].

Another drawback of this study may come from the fact that the PBI asks for evaluation of parental attitudes as well. Future study may make use of questionnaies such as the EMBU [49] that asks for frequencies of the parental behavior.

Taking these limitations into consideration, this study suggests that depression in undergraduate students is triggered by both depression-related cognitive styles and by poor perceived child rearing practices, and that the former mediates the effects of the latter.

\section{CONFLICT OF INTEREST}

The authors confirm that this article content has no conflicts of interest.

\section{ACKNOWLEDGEMENT}

None declared.

\section{REFERENCES}

[1] Beck AT. Cognitive therapy and emotional disorders. New York: International University Press 1976.

[2] Weissman A. The Dysfunctional Attitude Scale: A validation study. Dissertation Abstracts International, 1979; 40: 1389-90B. (University Microfilm No. 79-19, 533).

[3] Chioqueta AP, Stiles TC. Psychometric properties of the Norwegian version of the Dysfunctional Attitude Scale (Form A). Cogn Behav Ther 2004; 33: 83-6.

[4] Dobson KS, Shaw BF. Cognitive assessment with major depressive disorders. Cogn Ther Res 1986; 10: 13-29.
[5] Joiner TE Jr, Metalsky GI, Lew A, Klocek J. Testing the causal mediation component of Beck's theory of depression: evidence for specific mediation. Cogn Ther Res 1999; 23: 401-12.

[6] Kwon S, Oei TPS. Differential causal roles of dysfunctional attitudes and automatic thoughts in depression. Cogn Ther Res 1992; 16: 309-28.

[7] Olinger LJ, Kuiper NA, Shaw BF. Dysfunctional attitudes and stressful life events: an interactive model of depression. Cogn Ther Resh 1987; 11: 25-40.

[8] Robins CJ, Block P. Cognitive theories of depression viewed from a diathesis-stress perspective: Evaluations of the models of beck and of abramson, seligman, and teasdale. Cogn Ther Res 1989; 13: 297-313.

[9] Wierzbicki M, Bartlett TS. The efficacy of group and individual cognitive therapy for mild depression. Cogn Ther Res 1987; 11: 337-42.

[10] Wise EH, Barnes DR. The relationship among life events, dysfunctional attitudes, and depression. Cogn Ther Res 1986; 10: 257-66.

[11] Weich S, Churchill R, Lewis G. Dysfunctional attitudes and the common mental disorders in primary care. J Affect Disord 2003; 75: 269-78.

[12] Crandell CL, Chambless DL. The validation of an inventory for measuring depressive thoughts: the crandell cognitions inventory. Behav Res Ther 1986; 24: 403-11.

[13] Hollon SD, Kendall PC. Cognitive self-statements in depression: development of an automatic thoughts questionnaire. Cogn Ther Res 1980; 4: 383-95.

[14] Blackburn IM, Jones S, Lewin RJP. Cognitive style in depression. Br J Clin Psychol 1986; 25: 241-51.

[15] Dohr KB, Rush AJ, Bernstein IH. Cognitive biases and depression. J Abn Psychol 1989; 98: 263-7.

[16] Eaves G, Rush AJ. Cognitive patterns in symptomatic and remitted unipolar major depression. J Abn Psychol 1984; 93: 31-40.

[17] Hollon SD, Kendall PC, Lumry A. Specificity of depressotypic cognitions in clinical depression. J Abn Psychol 1986; 95: 52-9.

[18] Lam DH, Brewin CR, Woods RT, Bebbington PE. Cognition and social adversity in the depressed elderly. J Abn Psychol 1987; 96: 23-6.

[19] Harrell TH, Ryon NB. Cognitive-behavioral assessment of depression: clinical validation of the automatic thoughts questionnaire. $\mathrm{J}$ Consul Clin Psychol 1983; 51: 721-5.

[20] Kendall PC, Howard BL, Hays RC. Self-referent speech and psychopathology: the balance of positive and negative thinking. Cogn Ther Res 1989; 13: 583-98.

[21] Sahin NH, Shahin N. How dysfunctional are the dysfunctional attitudes in another culture? Br J Med Psychol 1992; 65: 17-26.

[22] Parker G. Parental 'Affectionless Control' as an antecedent to adult depression: a risk factor delineated. Arch Gen Psychiatry 1983; 40: 956-60.

[23] Burbach DJ, Kashani JH, Rosenberg TK. Parental bonding and depressive disorders in adolescents. J Child Psychol Psychiatry 1989; 30: 417-29.

[24] Kerver MJ, van Son MJ, de Groot PA. Predicting symptoms of depression from reports of early parenting: a one-year prospective study in a community sample. Acta Psychiat Scand 1992; 86: 26772.

[25] Parker G. Parental rearing style: Examining for links with personality vulnerability factors for depression. Soc Psychiatr Epidemiol 1993; 28: 97-100.

[26] Parker G, Kiloh L, Hayward L. Parental representations of neurotic and endogenous depressives. J Affect Disord 1987; 13: 75-82.

[27] Parker G, Hadzi-Paylovic D. Parental representations of melancholic and non-melancholic depressives: examining for specificity to depressive type and for evidence of additive effects. Psychol Med 1992; 22: 657-65.

[28] Mackinnon AJ, Henderson AS, Andrews G. Parental 'affectionless control' as an antecedent to adult depression: a risk factor refined. Psychol Med 1993; 23: 135-41.

[29] Martin G, Waite S. Parental bonding and vulnerability to adolescent suicide. Acta Psychiatr Scand 1994; 89: 246-54.

[30] Oakley-Browne MA, Joyce PR, Wells JE, Bushnell JA, Hornblow AR. Adverse parenting and other childhood experiences as risk factors for depression in women aged 18-44 years. J Affect Disord 1995; 34: 13-23. 
[31] Parker G, Hadzi-Pavlovic D, Greenwald S, Weissman M. Low parental care as a risk factor to lifetime depression in a community sample. J Affect Disord 1995; 33: 173-80.

[32] Plantes MM, Prousoff BA, Brennan J, Parker G. Parental representations of depressed outpatients from a USA sample. J Affect Disord 1988; 15: 149-55.

[33] Rey JM. Perceptions of poor maternal care associated with adolescent depression. J Affect Disord 1995; 34: 95-100.

[34] Rogers B. Reported parental behaviour and adult affective symptoms, 1. Associations and moderating factors. Psychol Med 1996a; 26: 51-61.

[35] Rogers B. Reported parental behaviour and adult affective symptoms, 2. Mediating factors. Psychol Med 1996b; 26: 63-77.

[36] Sato T, Sakado K, Uehara T, et al. Dysfunctional parenting as a risk factor to lifetime depression in a sample of employed Japanese adults: Evidence for the 'affectionless control' hypothesis. Psychol Med 1998; 28: 737-42.

[37] Sato T, Sakado K, Uehara T, Nishioka K, Kasahara Y. Perceived parental styles in a Japanese sample of depressive disorders: a replication outside Western culture. Br J Psychiatry 1997a; 170: 1735 .

[38] Sato T, Uehara T, Sakado K, et al. Dysfunctional parenting and a lifetime history of depression in a volunteer sample of Japanese workers. Acta Psychiatr Scand 1997b; 96: 306-310.

[39] Whisman MA, Kwon P. Parental representations, cognitive distortions, and mild depression. Cogn Ther Res 1992; 16: 557-568.

[40] Holmbeck GN. Toward terminological, conceptual, and statistical clarity in the study of mediators and moderators: Examples from the child-clinical and pediatric psychology literatures. J Consul Clin Psychol 1997; 65: 599-610.

[41] Radloff LS. The CES-D Scale: a self-report depression scale for research in the general population. Appl Psychol Meas 1977; 1: 385-401.

[42] Parker G, Tupling H, Brown LB. A parental bonding instrument. Br J Med Psychol 1979; 52: 1-10.

[43] Kitamura T, Suzuki T. A validation study of the Parental Bonding Instrument in a Japanese population. Jap J Psychiatry Neurol 1993; 47: 29-36.

[44] Holnbeck GN. Toward terminological, conceptual and statistical clarity in the study of mediators and moderators: examples from the child-clinical and pediatric psychology literatures. J Consul Clin Psychol 1997; 65: 599-610.

[45] Schermelleh-Engel K, Moosbrugger H, Müller H. Evaluating the fit of structural equation models: tests of significance and descriptive goodness-of-fit measures. Methods Psychol Res Online 2003; 8: 23-74.

[46] Gerlsma C Das J, Emmelkamp PMG. Depressed patients’ parental representations: stability across changes in depressed mood and specificity across diagnosis. J Affect Disord 1993; 27: 173-81.

[47] Richter J, Eisemann M, Richter G, Perris C. Determinants of recall of parental rearing behavior: the influence of age or loss of parents by separation or death. Psychopathology 1992; 25: 120-7.

[48] Ten J-Y, Roosa MW, Michaels M. Agreement between parent and child reports on parental behaviors. J Marr Fam 1994; 56: 341-55.

[49] Perris C, Jacobsson, L, Lindström H, von Knorring L, Perris H. Development of a new inventory for assessing memories of parental rearing behaviour. Acta Psychiatr Scand 1980; 61: 265-74.

Received: October 10, 2012 Revised: October 22, 2012

Accepted: October 22, 2012

(C) Kitamura and Tanaka Licensee Bentham Open.

This is an open access article licensed under the terms of the Creative Commons Attribution Non-Commercial License (http://creativecommons.org/licenses/ by-nc/3.0/) which permits unrestricted, non-commercial use, distribution and reproduction in any medium, provided the work is properly cited. 